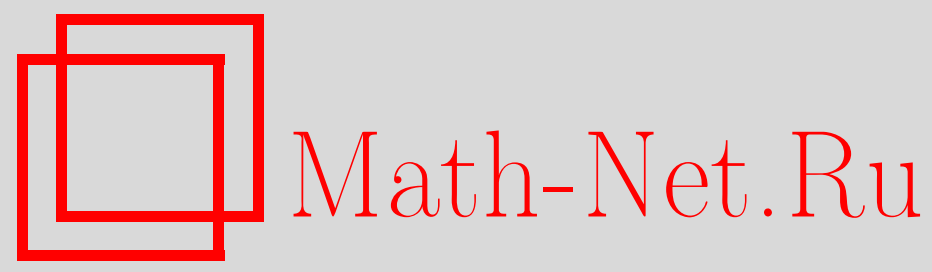

А. Ю. Зубов, K теоретико-игровому подходу исследования кодов аутентификации, Дискрет. матем., 2009, том 21, выпуск $3,45-72$

DOI: https://doi.org/10.4213/dm1061

Использование Общероссийского математического портала Math-Net.Ru подразумевает, что вы прочитали и согласны с пользовательским соглашением http://www . mathnet.ru/rus/agreement

Параметры загрузки:

IP: 54.84 .234 .179

26 апреля 2023 г., 15:02:02 
УДК 519.7

\title{
К теоретико-игровому подходу исследования кодов аутентификации
}

() 2009 г. А. Ю. Зубов

\begin{abstract}
Развивается теоретико-игровой подход к оценке стойкости кодов аутентификации, предложенный Г. Симмонсом. Доказывается, что комбинирование атак типа имитации и подмены может позволить злоумышленнику получить выигрыш, больший, чем выигрыш от атак типа имитации или подмены по отдельности.
\end{abstract}

\section{1. Введение}

В статье Г. Симмонса [3] предложен теоретико-игровой подход к исследованию математических моделей систем аутентификации, названных кодами аутентификации или $A$-кодами. Этот подход использует матричное представление $A$-кода и выбор оптимальных стратегий защиты и нападения с позиций теории игр. В последующих статьях, посвященных $A$-кодам (напр., $[2,4,6])$, стала активно использоваться теоретико-игровая терминология. Еще один важный теоретико-игровой аспект, отмеченный в указанной статье, состоит в том, что стойкость системы аутентификации определяется не только традиционными вероятностями успеха имитации или подмены, совпадающими со значениями $v_{i}$ и $v_{s}$ соответствующих матричных игр, но и возможностью комбинирования злоумышленником атак имитации и подмены, что соответствует игре с объединенной матрицей. Известно, что значение $v_{G}$ смешанного расширения такой игры удовлетворяет неравенству

$$
v_{G} \geqslant \max \left\{v_{i}, v_{s}\right\} .
$$

В [4] приведен пример $A$-кода с расщеплением, для которого в (1) выполняется строгое неравенство. Вместе с тем, в этом примере многое неясно, так как автор не дает никаких пояснений. Не представляется возможным проверить пример, поскольку неизвестны методы нахождения оптимальных стратегий для $A$-кодов, о чем заявляет в статье и сам автор. Не вызывает сомнения, что выяснение вопроса о том, выполняется ли для некоторого $A$-кода равенство или неравенство в (1), является важной задачей, поскольку именно величина $v_{G}$ является адекватной мерой стойкости $A$-кода к атакам на основе не более чем одного наблюдаемого сообщения. Сказанное свидетельствует об актуальности разработки методов вычисления оптимальных стратегий для $A$-кодов и значения объединенной игры. В данной статье проводится подробная теоретико-игровая формализация и предлагается метод вычисления оптимальной стратегии защиты в объединенной игре, учитывающей возможность комбинирования злоумышленником имитации и подмены. Приводится пример $A$-кода, для которого вычисляются значения соответствующих игр. Указываются достаточные условия, при выполнении которых стойкость $A$-кода оценивается максимумом вероятностей успеха имитации и подмены. 


\section{2. Основные определения}

В простейшей модели системы аутентификации информации принимают участие передатчик, приемник (сторона защиты) и оппонент (сторона нападения). Передатчик желает передать информацию о состоянии источника (которым может быть, например, результат подбрасывания монеты, или текстовое сообщение) приемнику по общедоступному каналу связи. С целью аутентификации передатчик образует сообщение, кодируя состояние источника с помощью правила кодирования, которое является секретным для оппонента. Используя то же правило, приемник проверяет аутентичность сообщения. Правило кодирования играет в этой модели роль секретного ключа, используемого при симметричном шифровании. В связи с этим должен быть предусмотрен комплекс мер по изготовлению и распределению секретных ключей передатчику и приемнику.

Оппонент имеет доступ к каналу связи и имеет полную информацию об используемой системе аутентификации, за исключением выбираемого стороной защиты правила кодирования. Оппонент имеет возможность осуществлять атаки имитации или подмены. Атака имитации состоит в попытке оппонента послать сообщение приемнику от имени передатчика, которое на самом деле передатчиком не посылалось. Атака подмены состоит в попытке оппонента подменить наблюдаемое им в канале связи сообщение другим сообщением, которое должно дезинформировать приемника о передаваемом состоянии источника. В обоих случаях оппонент рассчитывает на то, что поддельное сообщение будет принято как подлинное (аутентичное). Оппонент может выбирать вид атаки по своему усмотрению.

Отметим, что как имитация, так и подмена могут осуществляться оппонентом на основе наблюдения ряда сообщений в канале связи (как правило, полученных с помощью одного правила кодирования). Если оппонент принимает решение на основе наблюдения $r \geqslant 1$ сообщений, то говорят об активной атаке порядка $r$. В этой статье рассматривается лишь простейший тип атаки, когда оппонент принимает решение на основе наблюдения одного сообщения, при этом выбор поддельного сообщения при имитации не зависит от наблюдаемого сообщения (как в случае имитации в пустом канале).

Формальная модель системы аутентификации называется кодом аутентификации или $A$-кодом. Приведем соответствующие определения.

Пусть $S-$ (конечное) множество состояний источника, $M-$ (конечное) множество сообщений; сообщения передают информацию о состояниях источника; $E-$ (конечное) множество правил кодирования; правило кодирования $e \in E$ представляет собой инъективное отображение $e: S \rightarrow M$. Это отображение может быть многозначным. Тогда говорят о системе аутентификации с расщеплением. Если каждое правило кодирования является однозначным отображением, то говорят о системе аутентификации без расщепления. Введем для $e \in E$ и $m \in M$ обозначения

$$
\begin{aligned}
e(S) & =\{e(s): s \in S\}, \\
E(m) & =\{e \in E: m \in e(S)\} .
\end{aligned}
$$

Будем полагать, что выполняется равенство

$$
M=\bigcup_{e \in E} e(S),
$$

означающее, что каждое сообщение является результатом применения некоторого правила кодирования к некоторому состоянию источника. Каждое правило кодирования $e$ 
определяет обратное отображение $f_{e}(m): M \rightarrow S \cup\{0\}$ формулой

$$
f_{e}(m)= \begin{cases}s \in S, & \text { если } m=e(s), \\ 0, & \text { если } m \notin e(s) .\end{cases}
$$

При этом предполагается, что $0 \notin S$. Требование инъективности правила кодирования $e$ делает определение $f_{e}$ корректным. Для любых $s \in S$ и $e \in E$ выполняется равенство

$$
f_{e}(e(s))=s,
$$

означающее, что сообщение и выбранное правило кодирования однозначно восстанавливают состояние источника.

Определение 1. Тройка множеств $(S, E, M)$, где $S$ - множество состояний источника, $|S| \geqslant 2, M-$ множество сообщений, $|M| \geqslant 3, E-$ множество правил кодирования $e: S \rightarrow M,|E| \geqslant 3$, удовлетворяющих условиям (2) и (3), называется кодом аутентификации или $A$-кодом.

Передатчик и приемник для аутентификации передаваемых друг другу состояний источника выбирают (в секрете от оппонента) правило кодирования $e \in E$. Передатчик направляет приемнику сообщение $m=e(s)$. Критерием аутентичности полученного сообщения $m$ является условие $f_{e}(m) \neq 0$.

Отметим ряд требований, которые естественно предъявлять к любому $A$-коду. Из условия (2) следует неравенство $|M| \geqslant|S|$. В случае, если $|M|=|S|$, все сообщения будут приниматься приемником как аутентичные, и в этом случае аутентификация невозможна. Поэтому будем считать, что выполняется условие $|M|>|S|$.

Если некоторое сообщение, например, $m_{1}$, является допустимым лишь для одного правила кодирования $e \in E$, то есть, если $\left|E\left(m_{1}\right)\right|=1$, то, наблюдая $m_{1}$ и зная систему аутентификации, оппонент найдет $e$ и сможет подменить $m_{1}$ другим сообщением из множества $e(S)$, которое будет принято как аутентичное. Избежать этого позволяет условие, согласно которому каждое сообщение содержится во многих подмножествах аутентичных сообщений, то есть для любого $m \in M$

$$
\left|E\left(m_{1}\right)\right|>1
$$

С другой стороны, если выполняется равенство $E\left(m_{1}\right)=E$, то оппонент с вероятностью 1 добьется успеха, имитируя передачу $m_{1}$. Поэтому будем полагать, что для любого $m \in M$ выполняется неравенство

$$
|E(m)|<|E|
$$

Оппонент наверняка обманет, если заменит наблюдаемое сообщение $m$ другим сообщением из множества

$$
\bigcap_{e \in E(m)} e(S) .
$$

Поэтому естественно потребовать, чтобы для каждого $m \in M$ выполнялось условие

$$
\bigcap_{e \in E(m)} e(S)=\{m\} .
$$


Можно задать $A$-код матрицей кодирования размера $|E| \times|M|$, строки которой занумерованы правилами кодирования $e \in E$, столбцы сообщениями $m \in M$; на пересечении строки с номером $e$ и столбца с номером $m$ расположен элемент $f_{e}(m)$. Помимо матрицы кодирования вводится матрица $X_{A}$ инцидентности $A$-кода, имеющая те же размеры. Ее элементы $x(e, m)$ определяются формулой

$$
x(e, m)= \begin{cases}1, & \text { если } f_{e}(m) \neq 0, \\ 0, & \text { если } f_{e}(m)=0 .\end{cases}
$$

Условия (2) и (4)-(6) означают, что матрица $X_{A}$ не содержит нулевых строк и столбцов, содержит хотя бы один ноль и не менее двух единиц в каждой строке и каждом столбце, и не содержит двух и более столбцов, единицы одного из которых покрываются единицами другого.

Если в каждом столбце матрицы кодирования имеются вхождения лишь одного состояния источника, например, как в матрице

$$
\left(\begin{array}{cccc}
H & T & 0 & 0 \\
0 & T & H & 0 \\
H & 0 & 0 & T \\
0 & 0 & H & T
\end{array}\right)
$$

(содержащей два состояния источника: $H-$ орел, $T-$ решка), то такой $A$-код называется декартовым $A$-кодом или $A$-кодом без секретности, в противном случае $-A$-кодом с секретностью. Формально $A$-код без секретности характеризуется тем, что если $e(s)=m$, то для любого $e^{\prime} \in E(m)$ выполняется равенство $f_{e^{\prime}}(m)=s$. Другими словами, любое сообщение однозначно определяет состояние источника, поэтому передаваемое состояние источника не является секретом для оппонента.

Любой $A$-код без секретности можно представить в виде $A$-кода с аутентификатором. Так называются $A$-коды, для которых $M \subseteq S \times A$, где $A-$ множество аутентификаторов, и для любых $s \in S$ и $e \in E$ сообщение $m=e(s)$ имеет вид $(s, a)$ для подходящего $a \in A$. Другими словами, сообщение состоит из передаваемого состояния источника и добавленного к нему аутентификатора, вычисленного с помощью выбранного правила кодирования $e$. Аутентификатор является образом элемента $s$ при отображении $\bar{e}: S \rightarrow A$, таким образом, для $A$-кода с аутентификатором сообщение имеет вид $e(s)=(s, \bar{e}(s))$. Отображение $\bar{e}$ не обязано быть инъективным, поскольку при проверке аутентичности полученного сообщения $(s, a)$ нужно лишь вычислить значение $\bar{e}(s)$ и сравнить его с $a$. В связи с этим аутентификаторы могут быть достаточно короткими, при этом их длины определяются выбранным уровнем стойкости $A$-кода.

C $A$-кодом ассоциируется следующая тройка случайных величин. Предполагается, что состояния источника, правила кодирования и сообщения выбираются случайно. Пусть $\widetilde{S}-$ случайная величина, принимающая значения из $S$ и имеющая распределение $P(S)=\left(p_{S}(s), s \in S\right)$ при этом предполагается, что элементы множества $S$ некоторым естественным образом упорядочены. Тогда $P(S)$ рассматривается как вектор. Пусть $\widetilde{E}-$ случайная величина, принимающая значения из $E$ и имеющая распределение $P(E)=\left(p_{E}(e), e \in E\right)$. Распределение $P(S)$ считается заданным априорно и является неизменным, в то время как распределение $P(E)$ может регулироваться передатчиком и приемником с целью повышения надежности защиты от активных атак. Будем полагать, что состояния источника и правила кодирования выбираются независимо друг от друга, то есть, что случайные величины $\widetilde{S}$ и $\widetilde{E}$ являются независимыми. Они индуцируют случайную величину $\widetilde{M}$, принимающую значения из $M$ и имеющую распределение 
$P(M)=\left(p_{M}(m), m \in M\right)$. При этом вероятность $p_{M}(m)$ вычисляется для $A$-кода без расщепления (далее рассматриваются лишь такие $A$-коды) по формуле

$$
p_{M}(m)=\sum_{e \in E(m)} p_{E}(e) p_{S}\left(f_{e}(m)\right)
$$

которая следует из формулы полной вероятности

$$
p_{M}(m)=\sum_{e \in E} p_{E}(e) p_{M \mid E}(m \mid e)
$$

где

$$
p_{M \mid E}(m \mid e)= \begin{cases}p_{S}\left(f_{e}(m)\right), & \text { если } m \in e(S), \\ 0, & \text { если } m \notin e(S) .\end{cases}
$$

\section{3. Теоретико-игровая интерпретация $A$-кода}

Будем пользоваться общепринятыми в теории игр понятиями матричной игры, ситуации в матричной игре, функции выигрыша, чистых, смешанных и оптимальных стратегий (см., напр., [1]).

С $A$-кодом ассоциируются три матричные игры. В каждой из игр одна сторона нападает, а другая защищается. Будем далее, следуя принятой в теории игр терминологии, называть сторону нападения (оппонента) первым игроком, а сторону защиты (передатчика и приемника) вторым игроком.

При игре в имитацию ход первого игрока состоит в выборе сообщения $m \in M$, а ход второго - в выборе правила кодирования $e \in E$. Если $f_{e}(m) \neq 0$, то выигрывает первый игрок, если $f_{e}(m)=0$, то - второй. Чистая стратегия защиты соответствует выбору строки матрицы инцидентности $X_{A}$, а чистая стратегия нападения - выбору столбца. Значение функции выигрыша $\Phi_{i}$ в ситуации $(e, m)$ определяется равенством $P h i_{i}(e, m)=x(e, m)$.

Если игроки выбирают свои стратегии в игре случайно, в соответствии с некоторыми распределениями вероятностей $Q=Q(M)$ и $P=P(E)$, то говорят о смешанном расширении матричной игры; при этом $Q$ и $P$ называют, соответственно, смешанной стратегией нападения и смешанной стратегией защиты.

Согласно теореме о минимаксе [1], существуют оптимальные смешанные стратегии, которые минимизируют проигрыш стороны защиты и максимизируют выигрыш оппонента. Выигрыш оппонента в ситуации $(Q, P)$ выражается формулой

$$
v_{i}(Q, P)=\sum_{e \in E} \sum_{m \in M} p(e) x(e, m) q(m)=\sum_{m \in M} \sum_{e \in E(m)} p(e) q(m)
$$

Выигрыш, полученный при использовании игроками оптимальных смешанных стратегий, называется значением игры в имитацию и обозначается $v_{i}$. Таким образом, если $Q^{(0)}$ и $P^{(0)}-$ любая пара оптимальных смешанных стратегий, то

$$
v_{i}=v_{i}\left(Q^{(0)}, P^{(0)}\right) .
$$

Эта величина характеризует стойкость $A$-кода к атаке имитации. 
При рассмотрении игры в подмену заметим, что для $A$-кодов без расщепления из неравенств $n \neq m, f_{e}(n) \neq 0$ и $f_{e}(m) \neq 0$ следует, что $f_{e}(n) \neq f_{e}(m)$. Это означает, что при подмене наблюдаемого сообщения $m$ другим сообщением $n$, которое принимается как аутентичное, приемник будет дезинформирован о передаваемом состоянии источника. В этой игре второй игрок выбирает $(e, s), e \in E, s \in S$, а первый, наблюдая $m=e(s)$, выбирает сообщение $n \in M$, подменяющее $m$. Значение функции выигрыша в ситуации $((e, s), n)$ полагается равным

$$
y((e, s), n)= \begin{cases}1, & \text { если } e \in E(n), n \neq e(s), \\ 0 & \text { в противном случае. }\end{cases}
$$

Пусть $Y_{A}$ - матрица, составленная из элементов $y((e, s), n)$, строки которой пронумерованы парами $(e, s), e \in E, s \in E$, столбцы - сообщениями $n \in M$.

Смешанными стратегиями игроков служат соответственно распределение вероятностей $V=\{v(e, s), e \in E, s \in S\}$ на $E \times S$ и совокупность $U=\left\{U_{m}, m \in M\right\}$ распределений $U_{m}=\left(u_{m, n}, n \in M\right)$, где $u_{m, n}$ - вероятность выбора сообщения $n$, подменяющего $m$. Выигрыш $v_{\text {п }}(U, V)$ первого игрока в ситуации $(U, V)$ определяется средним значением выигрыша $y((e, s), n)$ при случайном выборе $(e, s) \in E \times S$ и $n \in M$ в соответствии с распределениями $V$ и $U$. Он выражается формулой

$$
\begin{aligned}
v_{\text {п }}(U, V) & =\sum_{e \in E} \sum_{s \in S} \sum_{n \in M} v(e, s) y((e, s), n) u_{e(s), n} \\
& =\sum_{e \in E} \sum_{s \in S} \sum_{n \in e(S)} v(e, s) u_{e(s), n}=\sum_{m \in M} \sum_{n \neq m} \sum_{e \in E(m, n)} v\left(e, f_{e}(m)\right) u_{m, n},
\end{aligned}
$$

где

$$
E(m, n)=E(m) \cap E(n)
$$

Нам понадобится и другая трактовка игры в подмену. Чтобы изложить ее, заметим, что вероятность $v(e, s)$ выбора вторым игроком пары $(e, s)$ (в силу независимости случайных величин $\widetilde{E}$ и $\widetilde{S}$ ) равна произведению вероятностей $p_{E}(e)$ и $p_{S}(s)$ :

$$
v(e, s)=p_{E}(e) p_{S}(s) .
$$

А так как распределение случайной величины $\widetilde{S}$ считается априорно заданным и не регулируется игроками, в смешанном расширении игры с матрицей $Y_{A}$ второй игрок фактически выбирает лишь распределение $P(E)$, которое можно считать смешанной стратегией защиты. Первый игрок должен выбрать сообщение, отличное от того, которое он будет наблюдать в канале связи. Это означает, что его ход состоит в выборе отображения $\varphi: M \rightarrow M$ без неподвижных точек. При этом любое наблюдаемое сообщение $m$ первый игрок заменит на $\varphi(m)$. Смешанная стратегия первого игрока - распределение вероятностей $R(\Psi)$ на множестве $\Psi$ отображений множества $M$ в себя без неподвижных точек.

Новая версия игры в подмену характеризуется матрицей $\bar{Y}_{A}$ размера $|E| \times|\Psi|$. Элемент $\bar{y}(e, \varphi)$ этой матрицы определяется как вероятность того, что в ситуации $(e, \varphi)$ модифицированное сообщение будет принято как аутентичное. Для вычисления $\bar{y}(e, \varphi)$ воспользуемся формулой полной вероятности. Пусть $A(e, \varphi)-$ событие, состоящее в успехе подмены в ситуации $(e, \varphi)$, и $A(e, \varphi, m)$ - событие, состоящее в успехе подмены 
наблюдаемого сообщения $m$ при данных $e, \varphi$. Тогда

$$
\begin{aligned}
\bar{y}(e, \varphi) & =\mathbf{P}\{A(e, \varphi)\}=\sum_{s \in S} \mathbf{P}\{A(e, \varphi, e(s))\} p_{S}(s) \\
& =\sum_{s \in S} y((e, s), \varphi(e(s))) p_{S}(s)=\sum_{s \in S: f_{e}(\varphi(e(s))) \neq 0} p_{S}(s) .
\end{aligned}
$$

Смешанными стратегиями в игре с матрицей $\bar{Y}_{A}$ служат соответственно распределения вероятностей $R(\Psi)=R=\left(r_{R}(\varphi) \varphi \in \Psi\right)$ и $P(E)=P$. Выигрыш первого игрока в ситуации $(R, P)$ выражается формулой

$$
\bar{v}_{\Pi}(R, P)=\sum_{e \in E} \sum_{\varphi \in \Psi} p_{E}(e) \bar{y}(e, \varphi) r_{R}(\varphi) .
$$

Для оптимальных смешанных стратегий $R^{(0)}, P^{(0)}$ получаем значение игры

$$
\bar{v}_{s}=\bar{v}_{s}\left(R^{(0)}, P^{(0)}\right) .
$$

Эта величина характеризует стойкость $A$-кода к атаке подмены.

Покажем, что обе версии игры в подмену эквивалентны, то есть имеют одинаковые значения и однозначно связанные друг с другом оптимальные стратегии защиты.

Обозначим через $\widehat{U}, \widehat{V}, \widehat{R}, \widehat{P}$ множества возможных стратегий $U, V, R, P$ соответственно.

Теорема 1. Игры в подмену с матрицами $Y_{A}$ и $\bar{Y}_{A}$ имеют одинаковые значения. Оптимальная стратегия зашиты в смешанных расширениях игры с матрицей $Y_{A}$ является оптимальной стратегией защитыь в расширении игры с матрицей $\bar{Y}_{A}$ и наоборот.

Доказательство. Отметим, прежде всего, что первая версия игры в подмену не является традиционной матричной игрой, поскольку смешанными стратегиями игроков служат не одномерные распределения вероятностей на множествах строк и столбцов матрицы игры, а двумерные распределения. В силу этого требуется доказательство аналога теоремы о минимаксе для этой версии игры, то есть наличие равенства

$$
\min _{V \in \widehat{V}} \max _{U \in \widehat{U}} v_{s}(U, V)=\max _{U \in \widehat{U}} \min _{V \in \widehat{V}} v_{s}(U, V)
$$

где $v_{s}(U, V)$ выражается формулой (11). Покажем, что это действительно так.

Пусть $R$ - смешанная стратегия нападения в игре с матрицей $\bar{Y}_{A}$. Обозначим через $U(R)$ смешанную стратегию нападения в игре с матрицей $Y_{A}$, индуцируемую стратегией $R$ по формуле

$$
u_{m, n}=\sum_{\varphi \in \Psi: \varphi(m)=n} r_{R}(\varphi) .
$$

Аналогично стратегия нападения $U$ в игре с матрицей $Y_{A}$ индуцирует стратегию нападения $R(U)$ в игре с матрицей $\bar{Y}_{A}$ по формуле

$$
r_{R}(\varphi)=\prod_{m \in M} u_{m, \varphi(m)}
$$


Несложно проверить, что $\left(u_{m, n}: n \in M\right)$ и $\left(r_{R}: \varphi \in \Psi\right)$, заданные формулами (17) и (18), образуют вероятностные распределения.

Покажем, что значение $v_{\text {п }}(U, V)$ игры с матрицей $Y_{A}$ в произвольной ситуации $(U, V)$ совпадает со значением $\bar{v}_{\text {п }}(R(U), V)$ игры с матрицей $\bar{Y}_{A}$ в ситуации $(R(U), V)$, где $R(U)$ определяется формулой (18), а распределения $V$ и $P$ связаны друг с другом формулой (12). В самом деле, подставляя выражение $u_{m, n}$ из (17) в (11) и используя (12), получаем равенства

$$
\begin{aligned}
v_{s}(U, P) & =\sum_{m \in M} \sum_{n \neq m} \sum_{e \in E(m, n)} \sum_{\varphi \in \Psi: \varphi(m)=n} p_{E}(e) p_{S}\left(f_{e}(m)\right) r_{R}(\varphi) \\
& =\sum_{m \in M} \sum_{\varphi \in \Psi} \sum_{e \in E(m, \varphi(m))} p_{E}(e) p_{S}\left(f_{e}(m)\right) r_{R}(\varphi) \\
& =\sum_{e \in E} \sum_{\varphi \in \Psi} \sum_{s \in S: f_{e}(\varphi(e(s))) \neq 0} p_{E}(e) p_{S}(s) r_{R}(\varphi) \\
& \left.=\sum_{e \in E} \sum_{\varphi \in \Psi} p_{E}(e) r_{R}(\varphi) \sum_{s \in S: f_{e}(\varphi(e(s))) \neq 0} p_{S}(s)=\bar{v}_{\Pi}(R(U), P)\right) .
\end{aligned}
$$

Покажем теперь, что значение $\bar{v}_{S}(R, P)$ игры с матрицей $\bar{Y}_{A}$ в произвольной ситуации $(R, P)$ совпадает со значением $v_{s}(U(R), P)$ игры с матрицей $Y_{A}$ в ситуации $(U(R), P)$, где $U(R)$ определяется формулой (17), а распределения $V$ и $P$ связаны друг с другом формулой (12). В самом деле, подставляя выражение $r_{R}(\varphi)$ из (18) в (14), получаем, что

$$
\begin{aligned}
\bar{v}_{\Pi}(R, P) & =\sum_{e \in E} \sum_{\varphi \in \Psi} p_{E}(e) \bar{y}(e, \varphi) r_{R}(\varphi)=\sum_{\varphi \in \Psi} r_{R}(\varphi) \sum_{e \in E} \sum_{s \in S: f_{e}(\varphi(e(s))) \neq 0} p_{E}(e) p_{S}(s) \\
& =\sum_{\varphi \in \Psi}\left(r_{R}(\varphi) \sum_{m \in M} \sum_{e \in E(m, \varphi(m))} p_{E}(e) p_{S}\left(f_{e}(m)\right)\right) \\
& =\sum_{\varphi \in \Psi}\left(\prod_{m^{\prime} \in M} u_{m^{\prime}, \varphi\left(m^{\prime}\right)} \sum_{m \in M} \sum_{e \in E(m, \varphi(m))} p_{E}(e) p_{S}\left(f_{e}(m)\right)\right) .
\end{aligned}
$$

Обозначим последнее выражение через $\Sigma$. Его можно представить в следующем виде:

$$
\begin{aligned}
& \Sigma=\sum_{\Delta(|M|)}\left(u_{m_{1}, n_{1}} \ldots u_{m_{|M|-1}, n_{|M|-1}} \cdot S_{|M|}\right)+\ldots \\
&+\sum_{\Delta(t)}\left(u_{m_{1}, n_{1}} \ldots u_{m_{t-1}, n_{t-1}} u_{m_{t+1}, n_{t+1}} \ldots u_{m_{|M|}, n_{|M|}} S_{t}\right) \\
&+\sum_{\Delta(1)}\left(u_{m_{2}, n_{2}} \ldots u_{\left.m_{|M|}, n_{|M|} S_{1}\right)},\right.
\end{aligned}
$$

где $\Delta(t)=\left\{\left(n_{1}, \ldots, n_{t-1}, n_{t+1}, \ldots n_{|M|}\right) \in M^{|M|-1}, n_{i} \neq m_{i}, i \in\{1, \ldots,|M|\} \backslash\{t\}\right\}$, a

$$
\left.S_{t}=\sum_{i \in\{1, \ldots,|M|\} \backslash\{t\}} u_{m_{t}, m_{i}} \sum_{j \in\{1, \ldots,|M|\} \backslash\{t\}} \sum_{e \in E\left(m_{j}, n_{j}\right)} p_{E}(e) p_{S}\left(f_{e}\left(m_{j}\right)\right)\right)
$$

при $t=1, \ldots,|M|$. Замечая теперь, что

$$
\sum_{i \in\{1, \ldots,|M|-1\}} u_{m_{|M|}, m_{i}}=\ldots=\sum_{i \in\{1, \ldots,|M|\} \backslash\{t\}} u_{m_{t}, m_{i}}=\ldots=\sum_{i \in\{2, \ldots,|M|\}} u_{m_{1}, m_{i}}=1,
$$


и производя в полученном выражении аналогичную группировку слагаемых, мы получим равенства

$$
\bar{v}_{\Pi}(R, P)=\sum_{m \in M} \sum_{n \neq m} \sum_{e \in E(m, n)} p_{E}(e) p_{S}\left(f_{e}(m)\right) u_{m, n}=v_{\Pi}(U(R), V) .
$$

Функция $v_{\text {п }}(U, V)$, определенная на компакте и принимающая значения из $[0,1]$, является непрерывной, и поэтому существуют минимакс и максимин, указанные в (16). Покажем, что они равны. Для этого заметим, что имеет место равенство

$$
\left\{v_{\Pi}(U, V): U \in \widehat{U}\right\}=\left\{v_{\Pi}(U(R), V): R \in \widehat{R}\right\},
$$

где $U(R)$ и $R$ связаны соотношением (17). В самом деле, включение $\supseteq$ очевидно. Обратное включение следует из цепочки равенств

$$
\left.v_{s}\left(U_{0}, V\right)=\bar{v}_{s}\left(R\left(U_{0}\right), P\right)\right)=v_{s}\left(\left(U\left(R_{0}\right), V\right)\right),
$$

где для любого $U_{0} \in \widehat{U}$ через $R_{0}$ обозначено распределение $R\left(U_{0}\right) \in \hat{R}$.

Из полученного равенства следует, что

$$
\max _{R \in \widehat{R}} \min _{P \in \widehat{P}} \bar{v}_{\Pi}(R, P)=\max _{R \in \widehat{R}} \min _{V \in \widehat{V}} v_{\Pi}(U(R), V)=\max _{U \in \widehat{U}} \min _{V \in \widehat{V}} v_{\Pi}(U, V) .
$$

Аналогично,

$$
\min _{P \in \widehat{P}} \max _{R \in \widehat{R}} \bar{v}_{\Pi}(R, P)=\min _{V \in \widehat{V}} \max _{R \in \widehat{R}} v_{\Pi}(U(R), V)=\min _{V \in \widehat{V}} \max _{U \in \widehat{U}} v_{\Pi}(U, V) .
$$

Поскольку вторая версия игры в подмену является обычной матричной игрой, для нее справедлива теорема о минимаксе, согласно которой

$$
\min _{P \in \widehat{P}} \max _{R \in \widehat{R}} \bar{v}_{\Pi}(R, P)=\max _{R \in \widehat{R}} \min \bar{v}_{\Pi \in \widehat{P}}(R, P)=\bar{v}_{\Pi} .
$$

Объединяя предыдущие соотношения, получаем (16). Полагая в (16) значение минимакса (и максимина) равным $v_{s}$, получаем попутно равенство $v_{s}=\bar{v}_{s}$. Теорема доказана.

Теорема 1 позволяет при нахождении значения игры и оптимальных смешанных стратегий использовать в зависимости от обстоятельств любую из приведенных трактовок игры в подмену. В связи с тем, что стратегия $V$ однозначно определяется стратегией $P$, будем далее вместо выражения $v_{s}(U, V)$ использовать выражение $v_{s}(U, P)$.

Приведем числовой пример. Рассмотрим $A$-код с множествами

$$
S=\{H, T\}, \quad M=\left\{m_{1}, m_{2}, m_{3}\right\}, \quad E=\left\{e_{1}, e_{2}, e_{3}\right\},
$$

распределением

$$
p_{S}(H)=p, \quad p_{S}(T)=1-p, \quad 0<p<1,
$$

и матрицей кодирования

$$
\left(\begin{array}{ccc}
H & T & 0 \\
T & 0 & H \\
0 & T & H
\end{array}\right)
$$


Для этого $A$-кода матрица инцидентности $X_{A}$ и матрица $Y_{A}$ имеют вид

$$
X_{A}=\left(\begin{array}{lll}
1 & 1 & 0 \\
1 & 0 & 1 \\
0 & 1 & 1
\end{array}\right), \quad Y_{A}=\left(\begin{array}{lll}
0 & 1 & 0 \\
1 & 0 & 0 \\
0 & 0 & 1 \\
1 & 0 & 0 \\
0 & 0 & 1 \\
0 & 1 & 0
\end{array}\right)
$$

Строки матрицы $Y_{A}$ пронумерованы, соответственно, парами $\left(e_{1}, H\right),\left(e_{1}, T\right),\left(e_{2}, T\right)$, $\left(e_{2}, H\right),\left(e_{3}, T\right),\left(e_{3}, H\right)$, а столбцы, соответственно, - символами $m_{1}, m_{2}, m_{3}$.

Матрица $\bar{Y}_{A}$ имеет вид

$$
\bar{Y}_{A}=\left(\begin{array}{cccccccc}
p & p & 1 & 1 & 0 & 0 & 1-p & 1-p \\
p & 0 & p & 0 & 1 & 1-p & 1 & 1-p \\
1-p & 1 & 0 & p & 1-p & 1 & 0 & p
\end{array}\right)
$$

Строки этой матрицы пронумерованы правилами кодирования $e_{1}, e_{2}, e_{3} \in E$, столбцы - отображениями $\varphi_{1}, \ldots, \varphi_{8}: M \rightarrow M$ без неподвижных точек, Каждое отображение соответствует стратегии нападения (подмены) и представляется двустрочной записью (подстановкой)

$$
\begin{array}{rlrl}
\varphi_{1} & =\left(\begin{array}{ccc}
m_{1} & m_{2} & m_{3} \\
2 & 3 & 1
\end{array}\right), & \varphi_{2} & =\left(\begin{array}{ccc}
m_{1} & m_{2} & m_{3} \\
2 & 3 & 2
\end{array}\right), \varphi_{3}=\left(\begin{array}{ccc}
m_{1} & m_{2} & m_{3} \\
2 & 1 & 1
\end{array}\right), \\
\varphi_{4} & =\left(\begin{array}{ccc}
m_{1} & m_{2} & m_{3} \\
2 & 1 & 2
\end{array}\right), \varphi_{5}=\left(\begin{array}{ccc}
m_{1} & m_{2} & m_{3} \\
3 & 3 & 1
\end{array}\right), \varphi_{6}=\left(\begin{array}{ccc}
m_{1} & m_{2} & m_{3} \\
3 & 3 & 2
\end{array}\right), \\
\varphi_{7} & =\left(\begin{array}{ccc}
m_{1} & m_{2} & m_{3} \\
3 & 3 & 1
\end{array}\right), \varphi_{8}=\left(\begin{array}{ccc}
m_{1} & m_{2} & m_{3} \\
3 & 1 & 2
\end{array}\right) .
\end{array}
$$

Как было отмечено ранее, оппонент (первый игрок) для достижения своих целей может выбирать между имитацией или подменой. Этой возможности отвечает игра с матрицей $Z_{A}$, являющейся конкатенацией матриц $X_{A}$ и $\bar{Y}_{A}$. Смешанное расширение $G$ игры с матрицей $Z_{A}$ назовем объединенной игрой. Стратегиями игроков в этой игре служат соответственно распределение вероятностей $P(E)=P$ (на множестве строк матрицы $Z_{A}$ ) и распределение вероятностей $W(M \cup \Psi)=W$ (на множестве столбцов матрицы $Z_{A}$ ). Значение $v_{G}$ объединенной игры - это вероятность выигрыша первого игрока в случае, когда игроки используют оптимальные стратегии $W^{(0)}$ и $P^{(0)}$. Эта величина является объективной мерой стойкости $A$-кода к рассматриваемым в этой статье простейшим атакам.

\section{4. Вероятности успеха атак имитации и подмены}

Случайный выбор состояния источника, правила кодирования и сообщения при имитации или подмене в соответствии с выбранными распределениями определяет вероятности успеха оппонента. Получим формулы для этих вероятностей в случае, когда стороны выбирают соответствующие распределения лучшим образом.

\section{1. Атака имитации}

Пусть $P(E)=P$ и $Q(M)=Q$ - смешанные стратегии защиты и нападения при имитации. Успех первого игрока при попытке имитации с помощью сообщения $m \in M$ 
определяется вероятностью события $m \in e(S)$ при случайном выборе $e \in E$. Обозначим эту вероятность через $p_{i}(m)$. Она выражается формулой

$$
p_{i}(m)=\sum_{e \in E(m)} p_{E}(e) .
$$

Если первый игрок случайно выбирает сообщение $m \in M$ в соответствии с распределением $Q(M)$, то среднее значение $p_{i}(m)$, равное $\sum_{m \in M} q(m) p_{i}(m)$, совпадает с величиной $v_{i}(Q, P)$, выраженной формулой (9). В зависимости от выбранных распределений, вероятность $v_{i}(Q, P)$, может изменяться в значительных пределах. Естественно, что второй игрок стремится минимизировать свои потери, а первый максимизировать свой выигрыш.

Второй игрок при выборе стратегии исходит из того, что первый игрок стремится получить максимальный выигрыш, равный $\max _{Q} v_{i}(Q, P)$. Лучшая стратегия защиты должна минимизировать этот проигрыш. В любом случае проигрыш не превзойдет $\min _{P} \max _{Q} v_{i}(Q, P)$. В свою очередь, первый игрок при выборе стратегии исходит из того, что второй игрок стремится обеспечить минимальный проигрыш, равный $\min _{P} v_{i}(Q, P)$. Выбор стратегии нападения должен максимизировать этот выигрыш. В любом случае выигрыш будет, не меньшим $\max _{Q} \min _{P} v_{i}(Q, P)$. Согласно теореме о минимаксе, указанные экстремумы существуют и равны, то есть справедливы равенства

$$
\min _{P} \max _{Q} v_{i}(Q, P)=\max _{Q} \min _{P} v_{i}(Q, P)=v_{i}\left(Q^{(0)}, P^{(0)}\right)=v_{i}
$$

где $Q^{(0)}$ и $P^{(0)}-$ любая пара оптимальных стратегий.

Получим удобное выражение для значения игры.

Пусть $P(E)=P$ - произвольная стратегия защиты и $m_{0}=m_{0}(P) \in M-$ сообщение, для которого выполняется равенство

$$
p_{i}\left(m_{0}\right)=\max _{m \in M} p_{i}(m)
$$

где $p_{i}(m)$ определяется формулой $(20)$. Пусть $\bar{Q}(P)=(\bar{q}(m), m \in M)-$ такая стратегия нападения, что

$$
\bar{q}(m)= \begin{cases}1, & \text { если } m=m_{0}, \\ 0, & \text { если } m \neq m_{0} .\end{cases}
$$

Теорема 2. Имеет место равенство

$$
v_{i}=\min _{P} \sum_{e \in E\left(m_{0}(P)\right)} p_{E}(e),
$$

где сообщение $m_{0}(P)$ определено условием (22).

Доказательство. Заметим, что указанная выше стратегия $\bar{Q}(P)$ дает максимальный выигрыш против стратегии $P$. В самом деле, если $Q-$ произвольная стратегия нападения, то справедливы соотношения

$$
\begin{aligned}
v_{\text {и }}(Q, P) & =\sum_{m \in M} q(m) p_{i}(m) \leqslant \sum_{m \in M} q(m) p_{i}\left(m_{0}\right)=p_{i}\left(m_{0}\right) \sum_{m \in M} q(m) \\
& =p_{i}\left(m_{0}\right)=\sum_{e \in E\left(m_{0}\right)} p_{E}(e)=v_{i}(\bar{Q}(P), P) .
\end{aligned}
$$


Пусть $Q^{(0)}, P^{(0)}$ - некоторая пара оптимальных стратегий. Тогда, используя полученное свойство стратегии $\bar{Q}(P)$, получаем соотношения

$$
v_{i}\left(\bar{Q}\left(P^{(0)}\right), P^{(0)}\right) \geqslant v_{i}\left(Q^{(0)}, P^{(0)}\right)=v_{i},
$$

из которых следует, что

$$
v_{i}\left(\bar{Q}\left(P^{(0)}\right), P^{(0)}\right)=v_{i}
$$

так как в противном случае стратегия $P^{(0)}$ позволяла бы получить выигрыш, больший, чем $v_{i}$, что противоречит условию ее оптимальности. Отсюда следует неравенство

$$
\min _{P} v_{i}(\bar{Q}(P), P) \leqslant v_{i}
$$

Предположим, что $\min _{P} v_{i}(\bar{Q}(P), P)<v_{i}$. Тогда найдется стратегия $P^{\prime}$, для которой $v_{i}\left(\bar{Q}\left(P^{\prime}\right), P^{\prime}\right)<v_{i}$. Воспользуемся тем, что использование оптимальной стратегии гарантирует первому игроку выигрыш, не меньший, чем значение игры. Это означает, что выполняется неравенство $v_{i}\left(Q^{(0)}, P^{\prime}\right) \geqslant v_{i}$. Отсюда получаем противоречивую цепочку неравенств

$$
v_{i} \leqslant v_{i}\left(Q^{(0)}, P^{\prime}\right) \leqslant v_{i}\left(\bar{Q}\left(P^{\prime}\right), P^{\prime}\right)<v_{i}
$$

Следовательно,

$$
v_{i}=\min _{P} v_{i}(\bar{Q}(P), P)
$$

откуда следует (24). Теорема доказана.

По определению вероятность успеха имитации совпадает со значением $v_{i}$ и определяется формулой (24). Обычно эту вероятность обозначают $p_{0}$.

Вычислим значение $v_{i}$ для приведенного выше примера $A$-кода. Для этого найдем явное выражение $\min _{p(E)} p_{\text {и }}\left(m_{0}\right)$, где $m_{0}$ определяется условием (22).

Пусть $p_{E}\left(e_{i}\right)=x_{i}, i \in\{1,2,3\}$. По матрице кодирования находим, что

$$
p_{i}\left(m_{1}\right)=x_{1}+x_{2}, \quad p_{i}\left(m_{2}\right)=x_{1}+x_{3}, \quad p_{i}\left(m_{3}\right)=x_{2}+x_{3} .
$$

Отсюда

$$
p_{i}\left(m_{0}\right)=\max _{m \in M} p_{\text {и }}(m)=\max \left\{x_{1}+x_{2}, x_{1}+x_{3}, x_{2}+x_{3}\right\} .
$$

Для нахождения $v_{i}$ нужно минимизировать $p_{i}\left(m_{0}\right)$ по множеству значений

$$
x_{1}+x_{2}+x_{3}=1, \quad 0<x_{i}<1 \text {. }
$$

Легко проверить, что

$$
L=\max \left\{x_{1}+x_{2}, x_{1}+x_{3}, x_{2}+x_{3}\right\} \geqslant 2 / 3,
$$

и что, если $x_{1}=x_{2}=x_{3}=1 / 3$, то $L=2 / 3$. Отсюда следует, что оптимальная стратегия защиты от имитации состоит в случайном равновероятном выборе $e \in E$, и $v_{i}=p_{0}=2 / 3$. Несложно также заметить, что для данного $A$-кода имеется лишь одна оптимальная стратегия защиты, причем она совпадает с единственной оптимальной стратегией нападения. 


\section{2. Атака подмены}

Успех оппонента при попытке подмены наблюдаемого сообщения $m \in M$ сообщением $n \neq m$ характеризуется вероятностью того, что для случайно выбранной пары $(e, s)$ происходит событие $A=\left\{n \neq m, f_{e}(n) \neq 0\right\}$ при условии, что произошло событие $B=\{e(s)=m\}$. Обозначим эту условную вероятность через $p_{s}(n \mid m)$. Она равна отношению вероятности одновременного наступления событий $A$ и $B$ к вероятности события $B$ :

$$
p_{s}(n \mid m)=\frac{1}{p_{M}(m)} \sum_{e \in E(m, n)} p_{E}(e) p_{S}\left(f_{e}(m)\right),
$$

где $p_{M}(m)$ определяется формулой (7). Стратегия нападения определяется совокупностью распределений $U=\left\{U_{m}, m \in M\right\}$, а стратегия защиты - распределением $P(E)=P$. При выбранных стратегиях $U$ и $P$ успех первого игрока определяется средним значением вероятности $p_{s}(n \mid m)$, то есть

$$
\sum_{m \in M} \sum_{n \neq m} p_{M}(m) p_{s}(n \mid m) u_{m, n}
$$

Из (7), (11), (26) и (27) следует, что среднее значение $p_{s}(n \mid m)$ совпадает с $v_{s}(U, P)$. Таким образом,

$$
v_{s}(U, P)=\sum_{m \in M} \sum_{n \neq m} p_{M}(m) p_{s}(n \mid m) u_{m, n} .
$$

Как и при имитации, значение $v_{s}$ игры в подмену определяется теоремой о минимаксе

$$
\min _{P} \max _{U} v_{s}(U, P)=\max _{U} \min _{P} v_{s}(U, P)=v_{s}\left(U^{(0)}, P^{(0)}\right)=v_{s} .
$$

Получим удобное выражение для $v_{s}$.

Пусть $P$ - произвольная стратегия защиты и $m^{0}=m^{0}(m, P) \in M-$ любое сообщение, определенное для данного $m \in M$ и распределения $P$ равенством

$$
p_{s}\left(m^{0} \mid m\right)=\max _{n \neq m} p_{s}(n \mid m) .
$$

Пусть $\bar{U}(P)=\bar{U}-$ стратегия защиты, состоящая из распределений

$$
\bar{U}_{m}=\left(\bar{u}_{m, m^{0}}=1, \bar{u}_{m, n}=0, n \neq m^{0}\right) .
$$

Теорема 3. Имеет место равенство

$$
v_{s}=\min _{P} \sum_{m \in M} \sum_{e \in E\left(m, m^{0}(m, P)\right)} p_{E}(e) p_{S}\left(f_{e}(m)\right),
$$

где сообщение $m^{0}(m, P)$ определено условием (30).

Доказательство. Нетрудно видеть, что указанная выше стратегия $\bar{U}(P)$ дает максимальный выигрыш против стратегии $P$. Точно так же, как в теореме 2 , доказывается равенство

$$
v_{s}=\min _{P} v_{s}(\bar{U}(P), P),
$$

из которого следует (32). 
По определению вероятность успеха подмены совпадает со значением $v_{s}$ и определяется формулой (32). Обычно эту вероятность обозначают $p_{1}$.

Вычислим значение $v_{s}$ для приведенного выше примера $A$-кода. Для этого найдем явное выражение суммы $\sum_{m \in M} p_{M}(m) p_{s}\left(m^{0} \mid m\right)$. Воспользуемся тем, что

$$
p_{M}(m) p_{s}\left(m^{0} \mid m\right)=\max _{n, n \neq m}\left(p_{M}(m) p_{s}(n \mid m)\right)=\max _{n, n \neq m} \sum_{e \in E(m, n)} p_{E}(e) p_{S}\left(f_{e}(m)\right) .
$$

С помощью матрицы кодирования находим, что

$$
\begin{aligned}
& p_{M}\left(m_{1}\right) p_{s}\left(m_{1}^{0} \mid m_{1}\right)=\max \left\{x_{1} p, x_{2}(1-p)\right\}, \\
& p_{M}\left(m_{2}\right) p_{s}\left(m_{2}^{0} \mid m_{2}\right)=\max \left\{x_{1}(1-p), x_{3}(1-p)\right\}, \\
& p_{M}\left(m_{3}\right) p_{s}\left(m_{3}^{0} \mid m_{3}\right)=\max \left\{x_{2} p, x_{3} p\right\},
\end{aligned}
$$

где, как и выше, $p_{E}\left(e_{i}\right)=x_{i}, i \in\{1,2,3\}$. Отсюда получаем, что

$$
L\left(x_{1}, x_{2}, x_{3}\right)=\max \left\{x_{1} p, x_{2}(1-p)\right\}+(1-p) \max \left\{x_{1}, x_{3}\right\}+p \max \left\{x_{2}, x_{3}\right\} .
$$

По определению

$$
v_{s}=p_{1}=\min _{P(E)} L\left(x_{1}, x_{2}, x_{3}\right) .
$$

Для нахождения $v_{s}$ нужно вычислить минимум $L\left(x_{1}, x_{2}, x_{3}\right)$ по множеству $\Delta$ значений переменных $x_{i}$, определяемому условиями (25). Это можно сделать путем вычисления $\min _{\Delta_{i}} L\left(x_{1}, x_{2}, x_{3}\right)$ в каждом из подмножеств $\Delta_{i}, i \in\{1,2, \ldots, 8\}$, множества $\Delta$, определяемых условиями достижения максимумов в трех слагаемых, составляющих (33). Рассмотрим, например, подмножество $\Delta_{7}$, определяемое неравенствами

$$
x_{1} p \leqslant x_{2}(1-p), \quad x_{1} \leqslant x_{3}, \quad x_{2} \geqslant x_{3} .
$$

Условия (34) можно записать в виде

$$
x_{1} \leqslant x_{3} \leqslant x_{2}, \quad x_{1} \leqslant x_{2}(1-p) / p .
$$

В условиях этого случая

$$
L\left(x_{1}, x_{2}, x_{3}\right)=x_{2}+x_{3}(1-p) .
$$

Из равенства $x_{1}+x_{2}+x_{3}=1$ и того, что в рассматриваемом случае $L\left(x_{1}, x_{2}, x_{3}\right)$ - линейная функция с положительными коэффициентами, не содержащая $x_{1}$, следует, что функция принимает минимальное значение при максимальном значении $x_{1}$. Из (35) найдем достижимую верхнюю оценку для $x_{1}$.

Если $p \leqslant 1 / 2$, то

$$
1=x_{1}+x_{2}+x_{3} \geqslant 3 x_{1},
$$

откуда $x_{1} \leqslant 1 / 3$. Если $x_{1}=1 / 3$, то $x_{1}=x_{2}=x_{3}$ и

$$
\min _{\Delta_{7}} L\left(x_{1}, x_{2}, x_{3}\right)=\frac{2-p}{3} .
$$

Если $p>1 / 2$, то

$$
1=x_{1}+x_{2}+x_{3} \geqslant x_{1}+x_{2} p /(p-1)+x_{1}=x_{1}(2-p)(1-p),
$$


откуда

$$
x_{1} \leqslant(2-p) /(1-p) .
$$

Если $x_{1}=(2-p) /(1-p)$, то

$$
x_{3}=x_{1}, \quad x_{2}=x_{1} p /(1-p)
$$

и

$$
\min _{\Delta_{7}} L\left(x_{1}, x_{2}, x_{3}\right)=\left(1-p+p^{2}\right) /(2-p) .
$$

Действуя аналогично в остальных случаях, получаем в итоге следующий результат: при $p \leqslant 1 / 2$

$$
\min L\left(x_{1}, x_{2}, x_{3}\right)=\min \left\{\frac{1-p+p^{2}}{1+p}, \frac{1-p^{2}}{2-p}, \frac{2-p}{3}\right\}=\frac{1-p^{2}}{2-p},
$$

а при $p>1 / 2$

$$
\min L\left(x_{1}, x_{2}, x_{3}\right)=\min \left\{\frac{1+p}{3}, \frac{p(2-p)}{1+p}, \frac{1-p+p^{2}}{2-p}\right\}=\frac{p(2-p)}{1+p} .
$$

Таким образом,

$$
v_{s}=p_{1}= \begin{cases}\left(1-p^{2}\right) /(2-p), & \text { если } 0<p \leqslant 1 / 2 \\ (p(2-p)) /(p+1), & \text { если } 1 / 2 \leqslant p<1\end{cases}
$$

Единственной оптимальной стратегий защиты от подмены является смешанная стратегия, определяемая распределением

$$
P^{(0)}(E)= \begin{cases}(1-p) /(2-p), p /(2-p),(1-p) /(2-p), & \text { если } 0<p \leqslant 1 / 2, \\ (1-p) /(1+p), p /(1+p), p /(1+p), & \text { если } 1 / 2 \leqslant p<1 .\end{cases}
$$

Единственность оптимальной стратегии защиты для данного $A$-кода позволяет полностью описать множество оптимальных стратегий нападения. В самом деле, любая оптимальная стратегия нападения $\bar{y}=\left(y_{1}, \ldots, y_{8}\right)$ удовлетворяет условию

$$
\bar{P}^{(0)}(E) \bar{Y}_{A} \bar{y}^{T}=v_{\Pi},
$$

откуда при $0<p \leqslant 1 / 2$ получаем уравнение

$$
\left(\frac{1-p}{2-p}, \frac{p}{2-p}, \frac{1-p}{2-p}\right) \bar{Y}_{A} \bar{y}^{T}=\frac{1-p^{2}}{2-p},
$$

а при $1 / 2 \leqslant p<1-$ уравнение

$$
\left(\frac{1-p}{1+p}, \frac{p}{1+p}, \frac{p}{1+p}\right) \bar{Y}_{A} \bar{y}^{T}=\frac{p(2-p)}{1+p} .
$$

Пользуясь матрицей (19), получаем эквивалентные выражения

$$
\frac{1-p+p^{2}}{2-p}\left(y_{1}+y_{3}+y_{5}+y_{7}\right)+\frac{1-p^{2}}{2-p}\left(y_{2}+y_{4}+y_{6}+y_{8}\right)=\frac{1-p^{2}}{2-p}
$$


и

$$
\frac{p(2-p)}{1+p}\left(y_{1}+y_{2}+y_{5}+y_{6}\right)+\frac{1-p+p^{2}}{1+p}\left(y_{3}+y_{4}+y_{7}+y_{8}\right)=\frac{p(2-p)}{1+p} .
$$

Отсюда следует, что при $0<p \leqslant 1 / 2$ множество оптимальных стратегий нападения совпадает с множеством решений уравнения

$$
y_{2}+y_{4}+y_{6}+y_{8}=1,
$$

а при $1 / 2 \leqslant p<1-$ с множеством решений уравнения

$$
y_{1}+y_{2}+y_{5}+y_{6}=1 \text {. }
$$

Сопоставляя результаты вычислений для данного $A$-кода значений $v_{i}$ и $v_{s}$, убеждаемся, что при $p=1 / 2$ оптимальной стратегией защиты, как от имитации, так и от подмены, является равновероятный выбор правил кодирования, а при $p \neq 1 / 2$ оптимальные стратегии защиты от имитации и от подмены различаются. Максимальное значение вероятности успеха подмены для рассмотренного $A$-кода равно $(4 \sqrt{3}-6) / 3 \approx 0,539$. Оно достигается для двух значений параметра $p$, именно, для $p=2-\sqrt{3}$ и $p=\sqrt{3}-1$.

\section{5. Объединенная игра}

Пусть

$$
G_{A}=\left(X_{A} \bar{Y}_{A}\right)=(z(e, \theta))_{e \in E, \theta \in M \cup \Psi},
$$

- матрица объединенной игры. Элемент $z(e, \theta)$ имеет вид

$$
z(e, \theta)= \begin{cases}x(e, m), & \text { если } \theta=m \in M \\ \bar{y}(e, \varphi), & \text { если } \theta=\varphi \in \Psi\end{cases}
$$

Пусть $v_{G}(W, P)$ - значение смешанного расширения объединенной игры в ситуации $(W, P)$, где $P=P(E)$ - стратегия защиты и $W=W(M \cup \Psi)-$ стратегия нападения. По определению,

$$
v_{G}(W, P)=\sum_{e \in E} \sum_{\theta \in M \cup \Psi} p(e) z(e, \theta) w(\theta)
$$

и значение $v_{G}$ игры выражается в виде

$$
v_{G}=v_{G}\left(W^{(0)}, P^{(0)}\right)=\max _{W} \min _{P} v_{G}(W, P),
$$

где $W^{(0)}$ и $P^{(0)}-$ оптимальные стратегии.

Стратегия $W$ естественным образом индуцирует стратегии имитации и подмены $Q_{W}$ и $R_{W}$. Пусть

$$
c=c(W)=\sum_{m \in M} w(m)
$$

тогда

$$
1-c=\sum_{\varphi \in \Psi} w(\varphi)
$$


Числа $c$ и $1-c$ представляют собой соответственно вероятности использования в стратегии $W$ первого игрока имитации и подмены. Распределения $Q_{W}$ и $R_{W}$ состоят из величин $q_{W}(m)=w(m) / c$ и $r_{W}(\varphi)=w(\varphi) /(1-c)$ соответственно. При этом значение объединенной игры в ситуации $(W, P)$ представляется в виде

$$
\begin{aligned}
v_{G}(W, P) & =\sum_{e \in E} \sum_{m \in M} p(e) z(e, m) w(m)+\sum_{e \in E} \sum_{\varphi \in \Psi} p(e) z(e, m) w(\varphi) \\
& =c \sum_{e \in E} \sum_{m \in M} p(e) x(e, m) q_{W}(m)+(1-c) \sum_{e \in E} \sum_{\varphi \in \Psi} p(e) \bar{y}(e, \varphi) r_{W}(\varphi) \\
& =c v_{\text {и }}\left(Q_{W}, P\right)+(1-c) v_{\text {п }}\left(R_{W}, P\right) .
\end{aligned}
$$

Как мы знаем из раздела 2 , стратегия подмены $R_{W}$ определяет стратегию подмены $U_{W}=U\left(R_{W}\right)$, состоящую из совокупности распределений $U m=\left(u_{m, n}, n \neq m\right)$, где

$$
u_{m, n}=\sum_{\varphi(m)=n} r_{W}(\varphi)=\sum_{\varphi(m)=n} \frac{w(\varphi)}{1-c} .
$$

Отсюда получаем выражения

$$
\begin{gathered}
v_{G}(W, P)=c v_{i}\left(Q_{W}, P\right)+(1-c) v_{s}\left(U_{W},\right. \\
v_{G}=v_{G}\left(W^{(0)}, P^{(0)}\right)=c^{(0)} v_{i}\left(Q_{W^{(0)}}, P^{(0)}\right)+\left(1-c^{(0)}\right) v_{s}\left(U_{W^{(0)}}, P^{(0)}\right),
\end{gathered}
$$

где $W^{(0)}, P^{(0)}$ - оптимальные стратегии в объединенной игре, $Q_{W(0)}$ и $U_{W}(0)-$ стратегии имитации и подмены, индуцируемые стратегией $W^{(0)}, c^{(0)}$ определяется формулой (40) при $W=W^{(0)}$.

Найдем удобное выражение для значения объединенной игры.

Теорема 4. Пусть $v_{G}=v_{G}\left(W^{(0)}, P^{(0)}\right)$ - значение объединенной игры, где $W^{(0)}, P^{(0)}$ - оптимальные смешанные стратегии. Пусть $Q_{W^{(0)}}$ и $U_{W^{(0)}}$ - смешанные стратегии имитации и подмены, индуцчируемые стратегией $W^{(0)}$. Тогда либо

$$
v_{G}=v_{i}=v_{i}\left(Q_{W^{(0)}}, P^{(0)}\right),
$$

либо

$$
v_{G}=v_{s}=v_{s}\left(U_{W^{(0)}}, P^{(0)}\right),
$$

либо

$$
v_{G}=v_{i}\left(Q_{W^{(0)}}, P^{(0)}\right)=v_{s}\left(U_{W^{(0)}}, P^{(0)}\right)
$$

Доказательство. Пусть в выражении (43) $c^{(0)}=1$. Отсюда получаем равенство $v_{G}=v_{i}\left(Q_{W^{(0)}}, P^{(0)}\right)$. Поскольку $c^{(0)}$ - это вероятность использования имитации в оптимальной стратегии нападения, равенство $c^{(0)}=1$ означает, что оптимальной стратегией первого игрока в объединенной игре является смешанная стратегия имитации. Эта стратегия гарантирует первому игроку выигрыш, равный $v_{G}$, и в игре в имитацию, поскольку $P^{(0)}$ можно рассматривать и как стратегию защиты в игре в имитацию. В самом деле,

$$
v_{G}=v_{G}\left(W^{(0)}, P^{(0)}\right)=v_{i}\left(Q_{W^{(0)}}, P^{(0)}\right) .
$$


Если бы для некоторой стратегии $P^{(1)}$ защиты от имитации выполнялось неравенство

$$
v_{i}\left(Q_{W^{(0)}}, P^{(0)}\right)>v_{i}\left(Q_{W^{(0)}}, P^{(1)}\right),
$$

мы имели бы соотношения

$$
v_{i}\left(Q_{W^{(0)}}, P^{(1)}\right)=v_{G}\left(W^{(0)}, P^{(1)}\right)<v_{G},
$$

которые противоречат оптимальности стратегии $P^{(0)}$ в объединенной игре. Следовательно, $\left(Q_{W^{(0)}}, P^{(0)}\right)$ - пара оптимальных стратегий в игре в имитацию, откуда следует (44). Аналогично при $c^{(0)}=0$ получаем (45).

Пусть теперь $c^{(0)} \notin\{0,1\}$. Если при этом $v_{i}\left(Q_{W^{(0)}}, P^{(0)}\right) \geqslant v_{s}\left(U_{W^{(0)}}, P^{(0)}\right)$, то из (43) следует, что $v_{G} \leqslant v_{i}\left(Q_{W^{(0)}}, P^{(0)}\right)$. Предположим, что $v_{G}<v_{i}\left(Q_{W^{(0)}}, P^{(0)}\right)$. Тогда рассмотрим смешанную стратегию нападения $\hat{Q}_{W}(0)$ в объединенной игре, определенную условием

$$
\hat{q}_{W^{(0)}}(\theta)= \begin{cases}q_{W^{(0)}}(m), & \text { если } \theta=m \in M, \\ 0, & \text { если } \theta=\varphi \in \Psi .\end{cases}
$$

Ясно, что

$$
v_{G}\left(\hat{Q}_{W^{(0)}}, P^{(0)}\right)=v_{S}\left(Q_{W^{(0)}}, P^{(0)}\right)
$$

Отсюда получаем неравенство $v_{G}<v_{G}\left(\hat{Q}_{W^{(0)}}, P^{(0)}\right)$, означающее, что стратегия $P^{(0)}$ дает первому игроку возможность получить больший выигрыш, чем $v_{G}$. Это противоречит условию оптимальности $P^{(0)}$. Следовательно, $v_{G}=v_{i}\left(Q_{W^{(0)}}, P^{(0)}\right)$, и

$$
v_{i}\left(Q_{W^{(0)}}, P^{(0)}\right)=c^{(0)} v_{i}\left(Q_{W^{(0)}}, P^{(0)}\right)+\left(1-c^{(0)}\right) v_{s}\left(U_{W^{(0)}}, P^{(0)}\right),
$$

откуда получаем (46).

К аналогичному заключению мы придем и в случае, если в (43) выполняется неравенство

$$
v_{i}\left(Q_{W^{(0)}}, P^{(0)}\right) \leqslant v_{s}\left(U_{W^{(0)}}, P^{(0)}\right) .
$$

Теорема доказана.

Приведенная теорема показывает, что если для $A$-кода выполняется неравенство

$$
v_{G}>\max \left\{v_{i}, v_{s}\right\}
$$

то оптимальную стратегию защиты в объединенной игре следует искать из равенства (46).

\section{6. Метод вычисления значения объединенной игры}

Сформулируем ряд следствий из теоремы 4.

Теорема 5. Если для A-кода выполняется неравенство (47), то

$$
v_{G}=v_{i}\left(\bar{Q}\left(P^{(0)}\right), P^{(0)}\right)=v_{s}\left(\bar{U}\left(P^{(0)}\right), P^{(0)}\right),
$$

где $P^{(0)}$ - оптимальная стратегия защчиты в объединенной игре, а $\bar{Q}\left(P^{(0)}\right)$ и $\bar{U}\left(P^{(0)}\right)$ - стратегии нападения, определяемые формулами (23) и (32) по стратегии $P^{(0)}$. 
Доказательство. По теореме 4, справедливы равенства (46).

Стратегия $\bar{Q}\left(P^{(0)}\right)$ такова, что

$$
v_{G}=v_{i}\left(Q_{W^{(0)}}, P^{(0)}\right) \leqslant v_{i}\left(\bar{Q}\left(P^{(0)}\right), P^{(0)}\right) .
$$

Но стратегия $P^{(0)}$ оптимальна и не может дать первому игроку выигрыш, превосходящий $v_{G}$. Следовательно,

$$
v_{G}=v_{i}\left(\bar{Q}\left(P^{(0)}\right), P^{(0)}\right) .
$$

Аналогично получаем равенство

$$
v_{G}=v_{s}\left(\bar{U}\left(P^{(0)}\right), P^{(0)}\right) .
$$

Теорема доказана.

Рассмотрим множество стратегий защиты в объединенной игре, удовлетворяющих уравнению

$$
v_{i}(\bar{Q}(P), P)=v_{s}(\bar{U}(P), P) .
$$

Теорема 6. Если для А-кода выполняется неравенство (47), то оптимальная стратегия зашиты $P^{(0)}$ в объединенной игре удовлетворяет равенству

$$
v_{G}=\min _{P} v_{i}(\bar{Q}(P), P),
$$

где минимум берется по всем стратегиям P, удовлетворяющчим условию (49).

Доказательство. Пусть стратегия защиты $P^{\prime}$ в объединенной игре удовлетворяет условию (49) и для нее достигается минимум в правой части равенства (50). Предположим, что $P^{\prime}$ не является оптимальной стратегией. Это означает, что она позволяет первому игроку получить выигрыш, превосходящий значение игры, то есть существует стратегия нападения $W$, такая, что

$$
v_{G}\left(W, P^{\prime}\right)>v_{G} .
$$

Согласно (42) для подходящей константы $c$ выполняется равенство

$$
v_{G}\left(W, P^{\prime}\right)=c v_{i}\left(Q_{W}, P^{\prime}\right)+(1-c) v_{s}\left(U_{W}, P^{\prime}\right) .
$$

Из свойства экстремальности стратегий $\bar{Q}(P)$ и $\bar{U}(P)$ следуют неравенства

$$
v_{i}\left(Q_{W}, P^{\prime}\right) \leqslant v_{i}\left(\bar{Q}\left(P^{\prime}\right), P^{\prime}\right), \quad v_{s}\left(U_{W}, P^{\prime}\right) \leqslant v_{s}\left(\bar{U}\left(P^{\prime}\right), P^{\prime}\right) .
$$

Поскольку $P^{\prime}$ удовлетворяет равенству (49), из (51)-(53) получаем соотношения

$$
\begin{aligned}
v_{G} & <v_{G}\left(W, P^{\prime}\right)=c v_{i}\left(\bar{Q}\left(P^{\prime}\right), P^{\prime}\right)+(1-c) v_{s}\left(\bar{U}\left(P^{\prime}\right), P^{\prime}\right) \\
& =c v_{i}\left(\bar{Q}\left(P^{\prime}\right), P^{\prime}\right)+(1-c) v_{\text {и }}\left(\bar{Q}\left(P^{\prime}\right), P^{\prime}\right)=v_{i}\left(\bar{Q}\left(P^{\prime}\right), P^{\prime}\right) .
\end{aligned}
$$

Пусть $P^{(0)}$ - оптимальная стратегия защиты в объединенной игре. Тогда, согласно теореме $5, P^{(0)}$ удовлетворяет равенству (48) и, в частности, условию (49). Тогда из (54) следует, что

$$
v_{i}\left(\bar{Q}\left(P^{(0)}\right), P^{(0)}\right)<v_{i}\left(\bar{Q}\left(P^{\prime}\right), P^{\prime}\right) .
$$

Мы получили противоречие с условием минимальности стратегии $P^{\prime}$. Отсюда следует, что стратегия $P^{\prime}$ оптимальна. Теорема доказана. 
Теорема 6 дает метод нахождения оптимальной стратегии защиты в объединенной игре в случае, когда выполняется условие (47). Для этого нужно решить уравнение (49) относительно $P$, а затем решить для полученного множества решений этого уравнения задачу минимизации функционала $v_{s}(\bar{Q}(P), P)$.

Теорема 7. Если для A-кода существует стратегия защиты $P$, являющаяся одновременно оптимальной при имитации и при подмене, то выполняется равенство

$$
v_{G}=\max \left\{v_{i}, v_{s}\right\}
$$

Доказательство. Пусть $P$ - стратегия, указанная в условии теоремы, и $W(P)-$ стратегия нападения, дающая первому игроку максимальный выигрыш против стратегии $P$ в объединенной игре. Согласно (42), для некоторого числа $c \in[0,1]$ выполняется равенство

$$
v_{G}(W(P), P)=c v_{i}(Q(P), P)+(1-c) v_{s}(U(P), P),
$$

где $Q(P)$ и $U(P)$ - соответственно стратегии нападения при имитации и при подмене, индуцируемые стратегией $W(P)$.

Учитывая, что стратегии $\bar{Q}(P)$ и $\bar{U}(P)$ дают первому игроку максимальный выигрыш против стратегии $P$ при имитации и при подмене соответственно, а также то, что $P-$ оптимальная стратегия защиты от обеих атак, получаем, что

$$
v_{i}(Q(P), P) \leqslant v_{i}(\bar{Q}(P), P)=v_{\text {и }}, \quad v_{s}(U(P), P) \leqslant v_{s}(\bar{U}(P), P)=v_{s} .
$$

Поскольку стратегия $P$ может быть неоптимальной в объединенной игре, из полученных соотношений следует, что

$$
v_{G} \leqslant v_{G}(W(P), P) \leqslant c v_{i}+(1-c) v_{s} \leqslant \max \left\{v_{i}, v_{s}\right\} .
$$

Искомое утверждение следует теперь из неравенства (1).

Аналогично доказывается следующее утверждение.

Теорема 8. Если для $A$-кода существует стратегия защить $P$, которая не дает оппоненту выцгрыш, больший чем $\varepsilon \in(0,1)$, как при имитации, так и при подмене, то $v_{G}<\varepsilon$.

Теоремы 7 и 8 обосновывают традиционно используемый подход к оценке стойкости $A$-кодов (см., напр., $[2,3,8])$, суть которого состоит в следующем. Доказывается, что вероятности $p_{0}, p_{1}$ успеха имитации и подмены для $A$-кода не превосходят выбранного порога $\varepsilon$, скажем, $\varepsilon=2^{-64}$. На основании этого делается заключение о стойкости $A$-кода к активным атакам. При этом, как правило, выбирается равновероятная стратегия защиты (как от имитации, так и от подмены), причем вероятность $p_{1}$ определяется не средним значением величины $p_{\text {п }}(m)=\max _{n \neq m} p_{\text {п }}(n \mid m)$, а максимумом $\bar{p}_{1}=\max _{m \in M} p_{\text {п }}(m)$. Поскольку при таком подходе справедливы неравенства

$$
v_{i}=p_{0} \leqslant \varepsilon, \quad v_{s} \leqslant p_{1} \leqslant \bar{p}_{1} \leqslant \varepsilon,
$$

выполняются условия теоремы 8 , согласно которой $v_{G}<\varepsilon$. Подчеркнем, что без теоремы 8 указанный подход был бы не достаточно обоснованным, так как требовалось бы формальное доказательство утверждения

$$
\left(p_{0} \leqslant \varepsilon, p_{1} \leqslant \varepsilon\right) \Rightarrow\left(v_{G} \leqslant \varepsilon\right) .
$$

Покажем, что существуют $A$-коды, для которых выполняется неравенство (47). 


\section{7. Пример}

Рассмотрим $A$-код, для которого

$$
\begin{gathered}
S=\{H, T\}, \quad E=\left\{e_{1}, e_{2}, e_{3}, e_{4},\right\}, \quad M=\left\{m_{1}, m_{2}, m_{3}, m_{4},\right\}, \\
P(S)=\left(p_{S}(H)=p, p_{S}(T)=1-p\right), \quad 1 / 2 \leqslant p<1,
\end{gathered}
$$

и матрица кодирования имеет вид

$$
\left(\begin{array}{cccc}
H & T & 0 & 0 \\
0 & H & T & 0 \\
0 & 0 & H & T \\
H & 0 & 0 & T
\end{array}\right)
$$

Пусть $P(E)=\left(x_{1}, x_{2}, x_{3}, x_{4}\right)-$ стратегия защиты.

Теорема 9. Для рассматриваемого А-кода справедливы равенства

$$
\begin{aligned}
v_{i} & =1 / 2, \\
v_{s} & =\frac{p}{2 p^{2}-p+1}, \\
v_{G} & =\frac{2 p}{2 p+1} .
\end{aligned}
$$

Оптимальные стратегии защиты в игре в имитащию, игре в подмену и в объединенной игре задаются соответственно распределениями

$$
\begin{aligned}
& x_{1}=1 / 4, \quad x_{2}=1 / 4, \quad x_{3}=1 / 4, \quad x_{4}=1 / 4 \text {, } \\
& x_{1}=x_{4}=\frac{p^{2}}{2 p^{2}-p+1}, \quad x_{2}=\frac{p(1-p)}{2 p^{2}-p+1}, \quad x_{3}=\frac{(1-p)^{2}}{2 p^{2}-p+1} \text {, } \\
& x_{1}=x_{3}=x_{4}=\frac{p}{2 p+1}, \quad x_{2}=\frac{1-p}{2 p+1} \text {. }
\end{aligned}
$$

В частности, при $p=1 / 2$

$$
v_{i}=v_{s}=v_{G}=1 / 2,
$$

причем равномерное распределение $P(E)$ является оптимальной стратегией защиты 8 любой игре.

Доказательство. Введем обозначение $a=(1-p) / p$. Пусть

$$
\begin{aligned}
L(\bar{x})= & \max \left\{x_{1}+x_{4}, x_{1}+x_{2}, x_{2}+x_{3}, x_{3}+x_{4}\right\}, \\
L_{1}(\bar{x})= & \max \left\{p x_{1} ; p x_{4}\right\}+\max \left\{(1-p) x_{1} ; p x_{2}\right\} \\
& \quad+\max \left\{(1-p) x_{2} ; p x_{3}\right\}+\max \left\{(1-p) x_{3} ;(1-p) x_{4}\right\} .
\end{aligned}
$$

Согласно определению,

$$
\begin{aligned}
v_{i} & =\min _{P(E)} L(\bar{x}), \\
v_{\text {п }} & =\min _{P(E)} L_{1}(\bar{x}),
\end{aligned}
$$


а согласно теореме 6 (в случае, если неравенство (47) действительно выполняется),

$$
v_{G}=\min _{\bar{x} \in \Omega} L(\bar{x}),
$$

где

$$
\Omega=\left\{\bar{x}: x_{1}+x_{2}+x_{3}+x_{4}=1, L(\bar{x})=L_{1}(\bar{x})\right\} .
$$

Формула (55) очевидна, причем ясно, что минимум $\min _{P(E)} L(\bar{x})$ достигается в случае, когда все $x_{i}$ одинаковы, то есть когда $P(E)$ - равномерное распределение. Также легко убедиться в том, что при $p=1 / 2$ и $\min _{P(E)} L_{1}(\bar{x})$ достигается при равенстве всех переменных, причем этот минимум равен $1 / 2$. Поэтому будем далее полагать, что выполняется условие $p>1 / 2$.

Вычислим минимум $\min _{\bar{x} \in \Delta_{i}} L_{1}(\bar{x})$ в каждом из подмножеств $\Delta_{i}, i \in\{1, \ldots, 16\}$, множества

$$
\Delta=\left\{\bar{x} \in \mathbf{R}^{4}: x_{1}>0, \ldots, x_{16}>0, \sum_{1}^{16} x_{i}=1\right\},
$$

определяемых условиями достижения максимума в слагаемых, составляющих $L_{1}(\bar{x})$. Попутно будем в каждом случае искать $\min _{\bar{x} \in \Omega_{i}} L(\bar{x})$, где $\Omega_{i}=\Omega \cap \Delta_{i}, i \in\{1, \ldots, 16\}$.

Случай 1. Область $\Delta_{1}$ определяется системой неравенств

$$
\begin{aligned}
x_{4} & \leqslant x_{1}, \\
p x_{2} & \leqslant(1-p) x_{1}, \\
p x_{3} & \leqslant(1-p) x_{2}, \\
x_{4} & \leqslant x_{3},
\end{aligned}
$$

которую можно переписать в виде

$$
x_{4} \leqslant x_{3} \leqslant a x_{2} \leqslant a^{2} x_{1} .
$$

В условиях этого случая

$$
L_{1}(\bar{x})=x_{1}+(1-p)\left(x_{2}+x_{3}\right) .
$$

Заметим, что $L_{1}-$ линейная форма с положительными коэффициентами, не содержащая $x_{4}$. Ясно, что она достигает минимума в случае, когда $x_{4}$ максимально возможно. Получим достижимую верхнюю оценку для $x_{4}$. Из (61) получаем соотношения

$$
1=x_{1}+x_{2}+x_{3}+x_{4} \geqslant \frac{1}{a^{2}} x_{4}+\frac{1}{a} x_{4}+2 x_{4}=\frac{2 p^{2}-3 p+2}{(1-p)^{2}} x_{4},
$$

из которых следует, что

$$
x_{4} \leqslant \frac{(1-p)^{2}}{2 p^{2}-3 p+2}
$$

Равенство в (62) достигается в том и только том случае, когда

$$
x_{4}=x_{3}=a x_{2}=a^{2} x_{1} .
$$


Отсюда

$$
x_{1}=\frac{p^{2}}{2 p^{2}-3 p+2}, \quad x_{2}=\frac{p(1-p)}{2 p^{2}-3 p+2}, \quad x_{3}=x_{4}=\frac{(1-p)^{2}}{2 p^{2}-3 p+2} .
$$

Вычисляя значение $L_{1}(\bar{x})$ на этом наборе значений переменных, получаем, что

$$
\min _{\bar{x} \in \Delta_{1}} L_{1}(\bar{x})=\frac{2 p^{2}-2 p+1}{2 p^{2}-3 p+2} .
$$

Для нахождения $\min _{\bar{x} \in \Omega_{1}} L(\bar{x})$ в условиях случая 1 рассмотрим 4 подслучая, определяемых условиями раскрытия максимума в выражении $L(\bar{x})$ :
(a) $\left\{\begin{array}{l}x_{2} \leq x_{4} \\ x_{3} \leq x_{1}\end{array}\right.$
(б) $\left\{\begin{array}{l}x_{4} \leqslant x_{2} \\ x_{3} \leqslant x_{1}\end{array}\right.$
(в) $\left\{\begin{array}{l}x_{4} \leqslant x_{2} \\ x_{1} \leqslant x_{3}\end{array}\right.$
(г) $\left\{\begin{array}{l}x_{2} \leqslant x_{4} \\ x_{1} \leqslant x_{3}\end{array}\right.$

В случае (а) $L(\bar{x})=x_{1}+x_{4}$. Из равенства

$$
L(\bar{x})=L_{1}(\bar{x})
$$

находим, что

$$
x_{4}=(1-p)\left(x_{2}+x_{3}\right) .
$$

Отсюда и из условий случая следует, что

$$
x_{4}=a x_{2} \leqslant x_{2} .
$$

Это противоречит условию $x_{2} \leqslant x_{4}$. Аналогично получаем противоречия в случаях $1(б)-$ 1(г). Таким образом, в случае 1 уравнение (64) не имеет решений.

Случай 2. Область $\Delta_{2}$ определяется системой неравенств

$$
\begin{aligned}
x_{4} & \leqslant x_{1}, \\
p x_{2} & \leqslant(1-p) x_{1}, \\
p x_{3} & \leqslant(1-p) x_{2}, \\
x_{4} & \geqslant x_{3} .
\end{aligned}
$$

В этих условиях

$$
L_{1}(\bar{x})=x_{1}+(1-p)\left(x_{2}+x_{4}\right) .
$$

Из условий случая можно получить достижимую верхнюю оценку для $x_{3}$ :

$$
1=x_{1}+x_{2}+x_{3}+x_{4} \geqslant \frac{1}{a^{2}} x_{3}+\frac{1}{a} x_{3}+2 x_{3}=\frac{2 p^{2}-3 p+2}{(1-p)^{2}} x_{3},
$$

и

$$
x_{3} \leqslant \frac{(1-p)^{2}}{2 p^{2}-3 p+2} .
$$

Отсюда получаем набор значений переменных, при котором $L_{1}$ достигает минимума, а также значение самого минимума. Они - те же, что в случае 1.

Для нахождения $\min _{\bar{x} \in \Omega_{2}} L(\bar{x})$ в условиях случая 2 рассмотрим 4 подслучая, указанных в (63). Легко проверить, что, как и в случае 1, каждый из этих подслучаев противоречив. Следовательно, в условиях случая 2 уравнение (64) не имеет решений. 
Случай 3. Область $\Delta_{3}$ определяется системой неравенств

$$
\begin{aligned}
x_{4} & \leqslant x_{1}, \\
p x_{2} & \leqslant(1-p) x_{1}, \\
p x_{3} & \geqslant(1-p) x_{2}, \\
x_{4} & \leqslant x_{3} .
\end{aligned}
$$

В условиях этого случая

$$
L_{1}(\bar{x})=x_{1}+x_{3}
$$

при этом

$$
x_{2} \leqslant \min \left\{a x_{1}, x_{4}\right\}, \quad x_{4} \leqslant \min \left\{x_{1}, x_{3}\right\} .
$$

Так как $L_{1}(\bar{x})$ - линейная функция, ее минимум достигается при $x_{1}=x_{3}=x_{4}=x_{2} / a$. Отсюда

$$
x_{1}=x_{3}=x_{4}=\frac{p}{2 p+1}, \quad x_{2}=\frac{1-p}{2 p+1},
$$

и

$$
\min _{\bar{x} \in \Delta_{3}} L_{1}(\bar{x})=\frac{2 p}{2 p+1} .
$$

Для нахождения $\min _{\bar{x} \in \Omega_{3}} L(\bar{x})$ в условиях случая 3 рассмотрим 4 подслучая, определяемых условиями (63).

В случае (a), $L(\bar{x})=x_{1}+x_{4}$. Из условий случая и равенства (64) следует, что

$$
x_{2} \leqslant \min \left\{a x_{1}, x_{3}=x_{4}\right\} \leqslant x_{1} .
$$

Минимальное значение $L(\bar{x})$ достигается при $x_{2}=x_{3}=x_{4}=a x_{1}$. Отсюда получаем, что

$$
\min _{\bar{x} \in \Omega_{3}} L(\bar{x})=\frac{1}{3-2 p},
$$

при этом значения переменных, при которых этот минимум достигается, равны

$$
x_{2}=x_{3}=x_{4}=\frac{1-p}{3-2 p}, \quad x_{1}=\frac{p}{3-2 p} .
$$

В случае (б), $L(\bar{x})=x_{1}+x_{2}$ и $x_{4} \leqslant x_{2}=x_{3} \leqslant a x_{1}$. Отсюда получаем те же значения, что и в предыдущем случае.

В случае (в), $L(\bar{x})=x_{2}+x_{3}$. Условия случая, а также равенство (64), приводят к противоречивым соотношениям $x_{1}=x_{2}<(1 / a) x_{2} \leqslant x_{1}$. Следовательно, в этом случае уравнение (64) не имеет решения.

В случае $(г), L(\bar{x})=x_{3}+x_{4}, x_{2} \leqslant x_{1}=x_{4} \leqslant x_{3}$ и $x_{2} \leqslant a x_{1}$. Отсюда получаем, что

$$
\min _{\bar{x} \in \Omega_{3}} L(\bar{x})=\frac{2 p}{2 p+1},
$$

при этом соответствующие значения переменных равны

$$
x_{1}=x_{3}=x_{4}=\frac{p}{2 p+1}, \quad x_{2}=\frac{1-p}{2 p+1} .
$$


В остальных случаях (за исключением случая 9), имеющего противоречивые условия $x_{1}<x_{4}<x_{3}<a x_{2}<a^{2} x_{1}$ ) определенных лексикографическим порядком следования условий раскрытия максимумов в выражении для $L_{1}(\bar{x})$, рассуждения совершенно аналогичны. Приведем необходимые сведения.

Подмножества $\Delta_{i}, i=1, \ldots, 16$, определяются системами неравенств вида

$$
\begin{array}{rl}
x_{4} & * x_{1}, \\
p x_{2} & *(1-p) x_{1}, \\
p x_{3} & *(1-p) x_{2}, \\
& x_{4} * x_{3},
\end{array}
$$

где вместо $*$ стоит знак $\leqslant$ или $\geqslant$. Поэтому для указания подмножества достаточно указать направления этих знаков неравенств. Так, например, случай, соответствующий подмножеству $\Delta_{1}$, получает обозначение $(<,<,<,<)$. Пользуясь такими обозначениями, укажем результаты для всех 16 случаев.

В случае $1(<,<,<,<)$ и случае $2(<,<,<,>)$

$$
\min _{\bar{x} \in \Delta_{i}} L_{1}(\bar{x})=\frac{2 p^{2}-2 p+1}{2 p^{2}-3 p+2}
$$

при

$$
x_{1}=\frac{p^{2}}{2 p^{2}-3 p+2}, \quad x_{2}=\frac{p(1-p)}{2 p^{2}-3 p+2}, \quad x_{3}=x_{4}=\frac{(1-p)^{2}}{2 p^{2}-3 p+2} .
$$

В случае $3(<,<,>,<)$, случае $4(<,<,>,>)$, случае $7(<,>,>,<)$, случае 8 $(<,>,>,>)$, случае $11(>,<,>,<)$ и случае $15(>,>,>,<)$

$$
\min _{\bar{x} \in \Delta_{i}} L_{1}(\bar{x})=\frac{2 p}{2 p+1}
$$

при

$$
x_{1}=x_{3}=x_{4}=\frac{p}{2 p+1}, \quad x_{2}=\frac{1-p}{2 p+1} .
$$

В случае $5(<,>,<,<)$, случае $6(<,>,<,>)$, случае $13(>,>,<,<)$ и случае 14 $(>,>,<,>)$

$$
\min _{\bar{x} \in \Delta_{i}} L_{1}(\bar{x})=\frac{1}{3-2 p}
$$

при

$$
x_{1}=x_{3}=x_{4}=\frac{1-p}{3-2 p}, \quad x_{2}=\frac{p}{3-2 p} .
$$

В случае $10(>,<,<,>)$, случае $12(>,<,>,>)$ и случае $16(>,>,>,>)$

$$
\min _{\bar{x} \in \Delta_{i}} L_{1}(\bar{x})=\frac{p}{2 p^{2}-p+1}
$$

при

$$
x_{1}=x_{4}=\frac{p^{2}}{2 p^{2}-p+1}, \quad x_{2}=\frac{p(1-p)}{2 p^{2}-p+1}, \quad x_{3}=\frac{(1-p)^{2}}{2 p^{2}-p+1} .
$$

Приводимые далее подслучаи определены соотношением (63). 
В случаях 3(а), 3(б), 4(а), 4(б), 7(а) и 13(в)

$$
\min _{\bar{x} \in \Omega_{i}} L(\bar{x})=\frac{1}{3-2 p}
$$

при

$$
x_{1}=\frac{p}{3-2 p}, \quad x_{2}=x_{3}=x_{4}=\frac{1-p}{3-2 p} .
$$

В случаях 5(б), 5(в), 6(б), 6(в), 7(в), 8(б), 8(в), 13(б), 14(б), 14(в), 15(б), 15(в), 16(б) и 16(в)

$$
\min _{\bar{x} \in \Omega_{i}} L(\bar{x})=\frac{1}{3-2 p}
$$

при

$$
x_{1}=x_{3}=x_{4}=\frac{1-p}{3-2 p}, \quad x_{2}=\frac{p}{3-2 p} .
$$

В случаях 3(г), 4(г), 7(г), 8(a), 8(г), 11(a), 11(г), 12(a), 12(г), 15(a), 15(г), 16(а) и 16(г)

$$
\min _{\bar{x} \in \Omega_{i}} L(\bar{x})=\frac{2 p}{2 p+1}
$$

при

$$
x_{1}=x_{3}=x_{4}=\frac{p}{2 p+1}, \quad x_{2}=\frac{1-p}{2 p+1} .
$$

В случаях 6(а) и 14(a)

$$
\min _{\bar{x} \in \Omega_{i}} L(\bar{x})=\frac{2 p}{2 p+1}
$$

при

$$
x_{1}=x_{2}=x_{4}=\frac{p}{2 p+1}, \quad x_{3}=\frac{1-p}{2 p+1} .
$$

В случаях 4(в), 5(а), 5(г), 6(г), 10(а)-(г), 11(в), 12(б), 12(в), 13(а), 13(г) и 14(г) уравнение (64) не имеет решений.

Пользуясь приведенными значениями, получаем, что

$$
\begin{aligned}
v_{\text {п }} & =\min \left\{\frac{2 p^{2}-2 p+1}{2 p^{2}-3 p+2}, \frac{1}{3-2 p}, \frac{2 p}{2 p+1}, \frac{p}{2 p^{2}-p+1}\right\} \\
& =\frac{p}{2 p^{2}-p+1}, \\
v_{G} & =\min \left\{\frac{1}{3-2 p}, \frac{2 p}{2 p+1}\right\}=\frac{2 p}{2 p+1} .
\end{aligned}
$$

Осталось заметить, что при вычислении значения $v_{G}$ мы корректно воспользовались теоремой 6. Для этого нужно доказать, что для рассматриваемого $A$-кода справедливо неравенство (47). Предположим, что для данного $A$-кода

$$
v_{G}=\max \left\{v_{\mathrm{и}}, v_{\Pi}\right\}=v_{\Pi}=\frac{p}{2 p^{2}-p+1} .
$$

Тогда, согласно теореме 4, выполняются равенства (45), причем оптимальной стратегией защиты $P^{(0)}$ в объединенной игре является оптимальная стратегия защиты от подмены. 
Вычислим значение игры в имитацию в ситуации $\left(Q\left(P^{(0)}\right), P^{(0)}\right)$. Для этого выпишем найденную в доказательстве оптимальную стратегию защиты от подмены:

$$
P^{(0)}=\left(\frac{p^{2}}{2 p^{2}-p+1}, \frac{p(1-p)}{2 p^{2}-p+1}, \frac{(1-p)^{2}}{2 p^{2}-p+1}, \frac{p^{2}}{2 p^{2}-p+1}\right) .
$$

Далее вычислим вероятности $p_{\text {и }}\left(m_{i}\right), i \in\{1,2,3,4\}$ :

$$
\begin{aligned}
& p_{\text {и }}\left(m_{1}\right)=x_{1}+x_{4}=\frac{2 p^{2}}{2 p^{2}-p+1}, \\
& p_{\text {и }}\left(m_{2}\right)=x_{1}+x_{2}=\frac{p}{2 p^{2}-p+1}, \\
& p_{\text {и }}\left(m_{3}\right)=x_{2}+x_{3}=\frac{1-p}{2 p^{2}-p+1}, \\
& p_{\text {и }}\left(m_{4}\right)=x_{3}+x_{4}=\frac{1-2 p+2 p^{2}}{2 p^{2}-p+1} .
\end{aligned}
$$

Теперь находим

$$
\max _{i \in\{1, \ldots, 4\}} p_{\text {и }}\left(m_{i}\right)=p_{\text {и }}\left(m_{1}\right)
$$

и

$$
Q\left(P^{(0)}\right)=(1,0,0,0)
$$

Таким образом,

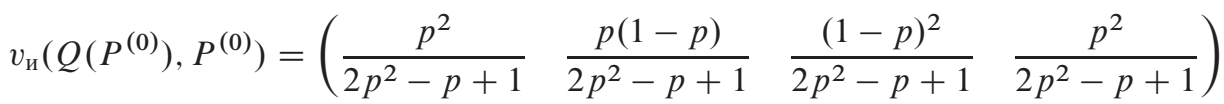

$$
\begin{aligned}
& \times\left(\begin{array}{llll}
1 & 1 & 0 & 0 \\
0 & 1 & 1 & 0 \\
0 & 0 & 1 & 1 \\
1 & 0 & 0 & 1
\end{array}\right)\left(\begin{array}{l}
1 \\
0 \\
0 \\
0
\end{array}\right)=\frac{2 p^{2}}{2 p^{2}-p+1}
\end{aligned}
$$

Поскольку

$$
\frac{2 p^{2}}{2 p^{2}-p+1}>\frac{p}{2 p^{2}-p+1}=v_{G}
$$

и $\left(Q\left(P^{(0)}\right), P^{(0)}\right)$ мы можем рассматривать как ситуацию в объединенной игре, получаем неравенство

$$
v_{G}\left(Q\left(P^{(0)}\right), P^{(0)}\right)>v_{G},
$$

которое противоречит оптимальности стратегии $P^{(0)}$ в объединенной игре. Это доказывает, что для данного $A$-кода выполняется условие (47).

Отметим, что при $p$, стремящемся к единице, значения $v_{s}$ и $v_{G}$ для данного $A$-кода стремятся соответственно к $1 / 2$ и $2 / 3$, так что разность $v_{G}-\max \left\{v_{i}, v_{s}\right\}$ стремится к $1 / 6$.

Автор выражает глубокую признательность А. М. Зубкову за ряд ценных замечаний и предложений, существенно улучшивших работу, а также С. В. Чернякову, который нашел с помощью ЭВМ пример из раздела 6 при конкретном значении параметра $p$. 


\section{Список литературы}

1. Воробьев Н. Н., Теория игр. Наука, Москва, 1985.

2. Den Boer B., A simple and key-economical unconditional authentication scheme. J. Computer Security (1993) 2, 65-71.

3. Johansson T., Kabatianskii G., Smeets B., On the relation between $A$-codes and codes correcting independent errors. Lect. Notes Computer Sci. (1994) 765, 1-11.

4. Simmons G. J., A game theoretical model of digital message authentication. Congressus Numerantium (1982) 34, 413-424.

5. Simmons G. J., Message authentication: A game on hypergraphs. Congressus Numerantium (1984) 45, 161-192.

6. Simmons G. J., Authentication theory/Coding theory. Lect. Notes Computer Sci. (1985) 196, 411-432.

7. Симмонс Г. Дж., Обзор методов аутентификации информации. ТИИЭР (1988) 76, №5, 105-125.

8. Stinson D. R., Universal hashing and authentication codes. Designs, Codes and Cryptography (1994) 4, 369-380.

Статья поступила 26.12.2007.

Переработанный вариант поступил 29.01.2008. 